\title{
COMPARACIÓN ENTRE LOS MOTIVOS PARA LA PRACTICA DE ACTIVIDAD FÍSICA SEGÚN EL GÉNERO Y EL TIEMPO DE PRÁCTICA EN UN GRUPO DE ESTUDIANTES
}

\author{
Azofeifa Mora, Christian Alberto \\ COMPARACION ENTRE LOS MOTIVOS PARA LA PRÁCTICA DE ACTIVIDAD FÍSICA SEGÚN EL GÉNERO Y \\ EL TIEMPO DE PRÁCTICA EN UN GRUPO DE ESTUDIANTES \\ MHSalud, vol. 14, núm. 2, 2018 \\ Universidad Nacional, Costa Rica \\ Disponible en: http://www.redalyc.org/articulo.oa?id=237054293001 \\ DOI: https://doi.org/10.15359/mhs.14-2.1
}

Esta obra está bajo una Licencia Creative Commons Atribución-NoComercial-SinDerivar 3.0 Internacional. 


\title{
COMPARACIÓN ENTRE LOS MOTIVOS PARA LA PRÁCTICA DE ACTIVIDAD FÍSICA SEGÚN EL GÉNERO Y EL TIEMPO DE PRÁCTICA EN UN GRUPO DE ESTUDIANTES
}

\author{
COMPARISON BETWEEN MOTIVATIONS TO PRACTICE PHYSICAL ACTIVITY BY \\ GENDER AND TIME OF PRACTICING THE ACTIVITY IN A GROUP OF STUDENTS \\ COMPARAÇÃO ENTRE OS MOTIVOS PARA A PRÁTICA DE ATIVIDADE FÍSICA \\ SEGUNDO O GẾNERO E O TEMPO DE PRÁTICA EM UM GRUPO DE ESTUDANTES
}

\author{
Christian Alberto Azofeifa Mora cristian.azofeifa.mora@una.cr \\ Universidad Nacional, Costa Rica
}

Recepción: 22 Febrero 2017

Aprobación: 10 Octubre 2017

DOI: https://doi.org/10.15359/mhs.14-2.1

Redalyc: http://www.redalyc.org/articulo.oa?id=237054293001

\begin{abstract}
Resumen:
El objetivo de este artículo es comparar los diferentes motivos para la práctica de ejercicio físico en un grupo de estudiantes universitarios de educación continua, según el género y el tiempo que llevan practicando ejercicio físico de forma constante. Para ello, se encuestaron 272 sujetos pertenecientes a distintos cursos libres relacionados con el ejercicio físico y temáticas afines. A los sujetos en estudio se les aplicó un cuestionario sociodemográfico (edad, género, tiempo realizando ejercicio de forma continua) y para la evaluación de los motivos para la práctica de ejercicio físico se utilizó el Autoinforme de motivos para la práctica de ejercicio físico (EMI-2 exercise motivations inventory). Los resultados indicaron de manera significativa $(p>.05)$ que las mujeres asocian aspectos relacionados con la salud corporal como motivos para la práctica de ejercicio físico en relación con los hombres; además, las personas que llevan más de un año realizando ejercicio de forma continua asocian de manera significativa $(p>.05)$ la afiliación y el reconocimiento social como sus principales motivos para la práctica de ejercicio físico en comparación con quienes llevan menor tiempo en la práctica. Se concluye que la mayor afinidad hacia la salud corporal por parte de las mujeres puede asociarse en mayor medida a percepciones de tipo social o personal, y los motivos de vinculo social y afiliación en las personas que llevan más tiempo realizando ejercicio físico se puede asociar a una mayor motivación hacia la pertenencia e interacción social.
\end{abstract}

Palabras clave:

Motivación, ejercicio, sexo, salud.

\section{Abstract:}

The objective of this paper is to compare the different motivations a group of university students has to practice physical exercise, based on their gender and the time they have regularly practiced such physical activity. For this purpose, 272 subjects taking different independent non-credit physical activity classes and/or related topics were surveyed. Subjects were given a sociodemographic questionnaire (age, gender, time regularly practicing the physical activity). The EMI-2 (Exercise Motivations Inventory-2) was used to assess motivations to practice physical activity. Results significantly indicated ( $p>.05$ ) that women relate body health as the motivation to practice physical activity. In addition, those who have regularly practiced the activity for over a year $(p>.05)$ relate affiliation and social recognition as their main motivations to practice physical activity compared to those who have less time practicing. It is concluded that women's greater affinity to body health may be mostly associated with social or personal perceptions, while the motivation of a social nature and affiliation in students who have practiced physical activity for longer may be related to a greater motivation towards a sense of belonging and social interaction.

Keywords:

motivation, exercise, sex, health. 


\section{Resumo:}

O objetivo deste artigo é comparar os diferentes motivos para a prática de exercício físico em um grupo de estudantes universitários de educação contínua, segundo o gênero e o tempo que levam praticando exercício físico de forma constante. Para isso, foram questionados 272 indivíduos pertencentes a distintos cursos livres relacionados com o exercício físico e temáticas afins. Foi aplicado um questionário sociodemográfico (idade, gênero, tempo realizando exercício de forma contínua) aos indivíduos em estudo e, para a avaliação dos motivos para a prática de exercício físico, foi utilizado o Autoinforme de motivos para a prática de exercício físico (EMI-2 exercise motivations inventory). Os resultados indicaram de maneira significativa $(p>.05)$ que as mulheres, em comparação aos homens, associam aspectos relacionados com a saúde corporal como motivos para a prática de exercício físico; além disso, as pessoas que levam mais de um ano realizando exercício de forma contínua associam de maneira significativa ( $p$ $>$.05) a afiliação e o reconhecimento social como seus principais motivos para a prática de exercício físico em comparação com aquelas que levam menor tempo na prática. Conclui-se que a maior afinidade com a saúde corporal por parte das mulheres pode estar associada em maior medida às percepções de tipo social ou pessoal e os motivos de vínculo social e afiliação nas pessoas que levam mais tempo realizando exercício físico pode estar associado a uma maior motivação à pertinência e interação social.

\section{Palavras-chave:}

Motivação, exercício, sexo, saúde.

\section{Introducción}

La práctica de ejercicio físico como hábito saludable dentro del rol de la cotidianidad implica, entre otros factores, importantes niveles de motivación que propicien su iniciación y continuidad a largo plazo. Asimismo, los mecanismos de adherencia al ejercicio son tan diversos en la población que asiste, ya sea de forma individual o grupal, a los distintos programas de ejercicio físico (Egli, Bland, Melton y Czech, 2011; Moreno-Collazos y Cruz-Bermúdez, 2015; Pérez-Muñoz y Sánchez-Vegas, 2015).

Dentro de los factores que motivan a los individuos a iniciar o mantenerse dentro de un régimen de ejercicio físico saltan a la luz una gran variedad asociados con importantes niveles de adhesión, que varían desde aspectos psicológicos, interpersonales, salud, imagen corporal o bien relacionados con la mejora de la aptitud física. De estos, la diversión, la afiliación, la mejora de la salud física actual y el estar con buenos niveles de condición física, se han reportado en estudios previos con mayor frecuencia (Folgar, Boubeta, Cristobal y Zamácola, 2012; Jiménez-Torres, Godoy-Izquierdo y Godoy-García, 2012; Navas y Soriano, 2016; Teixeira, Carraça, Markland, Silva y Ryan, 2012).

Aunado a lo anterior, diversas teorías han salido a la luz para tratar de explicar con mayor claridad los motivos reales para la práctica de ejercicio físico. Dentro de ellas, la Teoría de la Autodeterminación establece que los motivos que regulan la práctica de ejercicio físico pueden tener un origen intrínseco o extrínseco. Los motivos intrínsecos (factores internos del individuo) parecen tener un mayor peso de decisión al momento de realizar algún tipo de ejercicio físico, permitiendo una mayor adherencia a largo plazo. Entre estos, se asocian motivos relacionados con diversión, placer, desarrollo personal y satisfacción hacia las habilidades logradas. Por otro lado, la motivación, cuando proviene de factores más excéntricos (variables del entorno donde se realiza la actividad, premios, constructos sociales), se asocia con un menor peso motivacional en la continuidad de las personas hacia el ejercicio físico a largo plazo (Ball, Bice y Parry, 2014; Jiménez-Torres et al., 2012; Al Kubaisy, Mohamad, Ismail y Abdullah, 2015).

Aunado a lo anteriormente descrito, también coexiste la estructura tetradimensional de elementos motivacionales hacia la práctica de ejercicio físico, los cuales se asocian específicamente a la competencia, la condición física, el atractivo físico y la fuerza. Esta teoría es aceptada por diversos autores para explicar el arraigo hacia actividades relacionadas con el ejercicio físico (Navas y Soriano, 2016; Usán-Supervía, Salavera-Bordás, Murillo-Lorente y Megías-Abad, 2016)

Ahora bien, los motivos que acercan a las personas a la práctica de ejercicio físico pueden ser diversos en función de distintas variables como género o el tiempo que llevan realizando ejercicio de forma continua. Estudios previos han mostrado diferencias en los motivos hacia la práctica de ejercicio físico en función del género, con una predominancia asociada a la mejora de su apariencia corporal (perder grasa corporal, 
incrementar la masa muscular), cuya manifestación parece ser mayor en las mujeres debido a la presión cultural e interpersonal en comparación con los hombres (Guedes, Legnani y Legnani, 2013; Li et al., 2015; Tylka y Homan, 2015). Asimismo, factores de un carácter más extrínseco relacionados con la apariencia y una mayor preocupación con el control del peso corporal parece ser un motivo mayoritariamente manifestado por las mujeres, mientras que factores de un carácter más intrínseco como el incremento fuerza, el competir y la percepción del ejercicio como un reto, parece predominar en los hombres (Egli et al., 2011; Al Kubaisy et al., 2015; Louw, Van-Biljon y Mugandani, 2012; Molanorouzi, Khoo y Morris, 2015).

Por su parte, distintos niveles de participación indican diferentes motivaciones en la práctica física o deportiva. Por ejemplo, las personas con menor experiencia parecen centrar sus motivos en aspectos relacionados primordialmente con la imagen corporal, estética y otros aspectos físicos; por otra parte, en el caso de las personas con una experiencia intermedia, aspectos motivacionales como la aventura parecen tener una mayor influencia, y aquellos que poseen altos niveles de experiencia en dicha práctica suelen asociar sus motivos a aspectos ligados con la competición, las capacidades individuales y el placer, junto con aspectos asociados a las relaciones sociales (Azofeifa, 2006; Guedes et al., 2013; Ramírez et al., 2013). Asimismo, una posible hipótesis mencionada por Teixeira et al. (2012) afirma que aquellas personas carentes de un deseo o determinación verdadera hacia la práctica de ejercicio físico, a pesar de estar de manera activa en la realización de este, lo asocian más con sentimientos de "deber" que de "querer", por lo que se percibe más como un medio para lograr un fin y tales percepciones se ligan a la mejora de la apariencia física o el recibimiento de algún reconocimiento tangible. Esto es observado con mayor frecuencia en individuos que se inician en la práctica de ejercicio físico.

Por lo anteriormente descrito, el objetivo del presente estudio es analizar la relación entre los motivos hacia la práctica de ejercicio físico en un grupo de estudiantes universitarios según el género y el tiempo que llevan realizando ejercicio físico de manera continua.

\section{Metodología}

\section{Participantes}

La muestra fue intencionada y estuvo compuesta por 272 sujetos, los cuales se encontraban matriculados al momento de la recolección de datos en alguno de los cursos libres (educación continua) relacionados con diversos tópicos del ejercicio físico. Dicha muestra no constituye una población universitaria, esto debido a que la participación en tales cursos se ve únicamente sujeta al deseo de cada persona de matricularse en estos, tener concluida su educación secundaria y su respectivo pago por los aranceles relacionados con el costo del curso, lo cual hace que este sea un grupo poblacional muy diverso.

Sobre su composición, un 59,55 \% de la muestra estuvo representada por el sexo masculino y el restante $40,44 \%$ por el sexo femenino. La edad promedio de los hombres fue de $27 \pm 6,54$ años y la de las mujeres de $27 \pm 6,57$ años. Según el tiempo que llevan realizando ejercicio físico de forma continua, el $14 \%$ ha realizado ejercicio de forma continua menos de seis meses, el $11 \%$ ha realizado ejercicio entre seis meses a un año y el $75 \%$ reporta haber realizado ejercicio por más de un año.

\section{Instrumentos}

Para la obtención de las variables predictoras se aplicó un cuestionario sociodemográfico con los siguientes datos: edad, género y tiempo realizando ejercicio físico de forma continua. Además, se utilizó el Autoinforme de motivos para la práctica de ejercicio físico (EMI-2 exercise motivations inventory) que consiste en un cuestionario de autoinforme que busca evaluar el perfil motivacional del individuo para la práctica del ejercicio físico. Este cuestionario valora cinco áreas generales relacionadas con los motivos para la práctica de ejercicio físico (psicológicos, interpersonales, salud, relacionados con el cuerpo y de forma física. Consta de un total de 51 ítems y cada uno de ellos cuenta con una puntuación en escala continua de 0 a 10 puntos. Las puntuaciones obtenidas se clasifican en puntuaciones parciales, que son un total de 14 puntuaciones parciales las cuales representan el peso o importancia de cada tipo de motivo, según un valor de 0 a 10, y puntuaciones totales, que representan el peso o importancia de cada área general relacionada con la motivación, según un valor que puede oscilar entre 0 a 10 (Gutiérrez, Raich, Sánchez y Deus, 2003). 


\section{Procedimiento}

Para la obtención de los resultados, se les aplicó ambos cuestionarios, con su respectivo consentimiento informado, a los sujetos matriculados en los diferentes cursos libres relacionados con tópicos en las áreas del ejercicio físico (entrenamiento de la fuerza, entrenamiento funcional, Pilates, yoga, nutrición, entre otros). Estos cursos son matriculados por una población que presenta características muy variadas en cuanto a profesiones, oficios, sectores laborales donde se desempeñan y objetivos que los conducen a matricular dichos cursos, lo cual los convierte en una población muy heterogénea. Tales cursos son impartidos cada tres meses y tienen una duración de doce semanas.

La recolección de datos se realizó posterior a una explicación del objetivo de la investigación y fue realizada durante las primeras cuatro semanas de cada trimestre, durante el periodo del 2015 al 2016. Entre los criterios de inclusión a tomar en consideración para la participación de los sujetos en el estudio se consideraró que fueran mayores de edad (mínimo de 18 años), que se encontraran matriculados en algún curso libre ofertado durante cada trimestre y que no hubiesen ya participado en la encuesta en trimestres anteriores.

Una vez recolectados los datos durante el periodo determinado, se procedió a la clasificación de estos según los criterios de inclusión establecidos, para posteriormente proceder con su tabulación y respectivos análisis.

\section{Análisis estadístico}

Se aplicó estadística descriptiva e inferencial con análisis de varianza factorial para la determinación de la relación entre las variables predictoras y dependientes. Todos los análisis se realización con el paquete estadístico SPSS 24 para Microsoft Windows. 


\section{Resultados}

Tabla 1

Puntuación promedio entre los motivos para la práctica de ejercicio físico en un grupo de estudiantes de cursos libres según el género

\begin{tabular}{|c|c|c|c|}
\hline $\begin{array}{l}\text { Motivos Práctica } \\
\text { Ejercicio Físico }\end{array}$ & Género & $\mathbf{n}$ & Puntuación \\
\hline \multirow{2}{*}{ Vigor } & Masculino & 162 & $7,43 \pm 3$ \\
\hline & Femenino & 110 & $7,94 \pm 2,9$ \\
\hline \multirow{2}{*}{ Diversión } & Masculino & 162 & $7,83 \pm 2,96$ \\
\hline & Femenino & 110 & $7,95 \pm 2,74$ \\
\hline \multirow{2}{*}{ Desafio } & Masculino & 162 & $6,98 \pm 3,35$ \\
\hline & Femenino & 110 & $6,93 \pm 2,91$ \\
\hline \multirow{2}{*}{ Reconocimiento social } & Masculino & 162 & $4,01 \pm 2,9$ \\
\hline & Femenino & 110 & $2,84 \pm 2,63$ \\
\hline \multirow{2}{*}{ Afiliación } & Masculino & 162 & $4,9 \pm 3,1$ \\
\hline & Femenino & 110 & $4,82 \pm 3,17$ \\
\hline \multirow{2}{*}{ Competición } & Masculino & 162 & $5,35 \pm 3,44$ \\
\hline & Femenino & 110 & $3,66 \pm 2,98$ \\
\hline \multirow{2}{*}{ Urgencias de salud } & Masculino & 162 & $3,83 \pm 2,91$ \\
\hline & Femenino & 110 & $3,97 \pm 3,04$ \\
\hline \multirow{2}{*}{ Prevención } & Masculino & 162 & $7,54 \pm 3,19$ \\
\hline & Femenino & 110 & $8,24 \pm 2,69$ \\
\hline \multirow{2}{*}{ Salud corporal* } & Masculino & 162 & $7,79 \pm 3,4$ \\
\hline & Femenino & 110 & $8,46 \pm 2,73$ \\
\hline \multirow{2}{*}{ Control de peso } & Masculino & 162 & $5,88 \pm 3,24$ \\
\hline & Femenino & 110 & $6,72 \pm 3,01$ \\
\hline \multirow{2}{*}{ Imagen corporal } & Masculino & 162 & $6,29 \pm 3$ \\
\hline & Femenino & 110 & $6,28 \pm 2,88$ \\
\hline \multirow{2}{*}{ Fuerza y resistencia } & Masculino & 162 & $7,66 \pm 3,18$ \\
\hline & Femenino & 110 & $7,96 \pm 2,59$ \\
\hline \multirow{2}{*}{ Agilidad y flexibilidad } & Masculino & 162 & $6,55 \pm 3,36$ \\
\hline & Femenino & 110 & $7,2 \pm 2,89$ \\
\hline
\end{tabular}

$* P=.002$

En la tabla 1 se observan las puntuaciones promedio para cada uno de los motivos para la práctica de ejercicio físico según el género, los cuales demostraron que después de un análisis comparativo existen diferencias estadísticamente significativas $(T=-1,802$; sig $=.002)$ entre hombres y mujeres. Específicamente, la puntuación promedio para los motivos relacionados con la salud corporal fue significativamente mayor en las mujeres $(8,46 \pm 2,73)$ en comparación con los hombres $(7,79 \pm 3,4)$. 
Tabla 2

Puntuación promedio entre los motivos para la práctica de ejercicio físico en un grupo de estudiantes de cursos libres según el tiempo que llevan realizando ejercicio físico de forma continua

\begin{tabular}{|c|c|c|c|}
\hline Motivos & Tiempo realizando ejercicio & $\mathbf{n}$ & Puntuación Promedio \\
\hline \multirow{3}{*}{ Control del estrés } & Menos de 6 meses & 39 & $6,84 \pm 1,98$ \\
\hline & De 6 meses a 1 año & 31 & $6,64 \pm 3,08$ \\
\hline & Más de 1 año & 202 & $6,81 \pm 2,84$ \\
\hline \multirow{3}{*}{ Vigor } & Menos de 6 meses & 39 & $8,38 \pm 1,78$ \\
\hline & De 6 meses a 1 año & 31 & $7,38 \pm 3,09$ \\
\hline & Más de 1 año & 202 & $7,53 \pm 3,11$ \\
\hline \multirow{3}{*}{ Diversion } & Menos de 6 meses & 39 & $7,43 \pm 2,07$ \\
\hline & De 6 meses a 1 año & 31 & $7,67 \pm 3,17$ \\
\hline & Más de 1 año & 202 & $8 \pm 2,96$ \\
\hline \multirow{3}{*}{ Desafio } & Menos de 6 meses & 39 & $6,97 \pm 3,28$ \\
\hline & De 6 meses a 1 año & 31 & $6,41 \pm 3,43$ \\
\hline & Más de 1 año & 202 & $7,04 \pm 3,12$ \\
\hline \multirow{3}{*}{ Reconocimiento social } & Menos de 6 meses & 39 & $2,79 \pm 2,76$ \\
\hline & De 6 meses a 1 año & 31 & $2,67 \pm 2,5$ \\
\hline & Más de 1 año* & 202 & $3,82 \pm 2,88$ \\
\hline \multirow{3}{*}{ Afiliacion } & Menos de 6 meses & 39 & $4,23 \pm 2,76$ \\
\hline & De 6 meses a 1 año & 31 & $3,67 \pm 3,12$ \\
\hline & Más de 1 año* & 202 & $5,18 \pm 3,14$ \\
\hline \multirow{3}{*}{ Competicion } & Menos de 6 meses* & 39 & $3,66 \pm 2,72$ \\
\hline & De 6 meses a 1 año & 31 & $4,29 \pm 3,34$ \\
\hline & Más de 1 año & 202 & $4,92 \pm 3,45$ \\
\hline \multirow{3}{*}{ Urgencias de salud } & Menos de 6 meses & 39 & $4,12 \pm 2,95$ \\
\hline & De 6 meses a 1 año & 31 & $3,48 \pm 2,43$ \\
\hline & Más de 1 año & 202 & $3,91 \pm 3,04$ \\
\hline \multirow{3}{*}{ Prevención } & Menos de 6 meses & 39 & $8,79 \pm 1,88$ \\
\hline & De 6 meses a 1 año & 31 & $7,35 \pm 3,2$ \\
\hline & Más de 1 año & 202 & $7,69 \pm 3,13$ \\
\hline \multirow{3}{*}{ Salud corporal } & Menos de 6 meses & 39 & $8,71 \pm 2,32$ \\
\hline & De 6 meses a 1 año & 31 & $8,06 \pm 3,34$ \\
\hline & Más de 1 año & 202 & $7,93 \pm 3,27$ \\
\hline \multirow{3}{*}{ Control de peso } & Menos de 6 meses & 39 & $7,1 \pm 2,82$ \\
\hline & De 6 meses a 1 año & 31 & $5,77 \pm 3,12$ \\
\hline & Más de 1 año & 202 & $6,12 \pm 3,22$ \\
\hline \multirow{3}{*}{ Imagen corporal } & Menos de 6 meses & 39 & $6,64 \pm 2,14$ \\
\hline & De 6 meses a 1 año & 31 & $5,61 \pm 3,02$ \\
\hline & Más de 1 año & 202 & $6,32 \pm 3,06$ \\
\hline \multirow{3}{*}{ Fuerza y resistencia } & Menos de 6 meses & 39 & $8,28 \pm 1,97$ \\
\hline & De 6 meses a 1 año & 31 & $7,38 \pm 3,33$ \\
\hline & Más de 1 año & 202 & $7,74 \pm 3,05$ \\
\hline \multirow{3}{*}{ Agilidad y flexibilidad } & Menos de 6 meses & 39 & $7,33 \pm 2,47$ \\
\hline & De 6 meses a 1 año & 31 & $6,67 \pm 3,23$ \\
\hline & Más de 1 año & 202 & $6,74 \pm 3,31$ \\
\hline
\end{tabular}

${ }^{*} P<.05$

En la tabla 2 se observan las puntuaciones promedio para cada uno de los motivos para la práctica de ejercicio físico según el tiempo que se lleva realizando ejercicio físico de forma regular. Estos resutados evidencian diferencias estadísticamente significativas para los motivos relacionados con el reconocimiento social $(F=3.803$; sig $=.024)$ y la afiliación $(F=4.178 ;$ sig $=.016)$. De acuerdo con los resultados del post-hoc de Bonferrony, en el caso de los motivos relacionados con el reconocimiento social es estadísticamente superior en las personas que llevan más de un año realizando ejercicio de 
forma regular, en comparación con aquellos que llevan tanto de seis meses a un año o los que llevan menos de seis meses realizando ejercicio físico regularmente. Para el caso de los motivos relacionados con la afiliación, el mismo post-hoc demostró a nivel estadístico que conforme aumenta el tiempo realizando ejercicio de forma regular, los motivos relacionados con la afiliación fueron mayores.

\section{Discusión}

Las diversas razones o motivos que conducen a las personas a iniciar y mantenerse dentro de algún régimen o programa de ejercicio físico pueden ser tan variados como la diversidad de ofertas. Los motivos pueden asociarse con aspectos de figura o forma corporal, salud, diversión, afiliación, entre otros (Moreno-Collazos y Cruz-Bermúdez, 2015; Navas y Soriano, 2016; Pérez-Muñoz y Sánchez-Vegas, 2015). Ante ello, el objetivo del presente estudio fue analizar la relación entre los motivos hacia la práctica de ejercicio físico en un grupo de estudiantes universitarios de educación abierta según el género y el tiempo que llevan realizando ejercicio físico de manera continua.

Al comparar el género con los diversos motivos hacia la práctica de ejercicio físico, las mujeres mostraron puntuaciones significativamente mayores hacia una mayor asociación de la práctica de ejercicio físico con motivos relacionados con la salud corporal en comparación con los hombres. Tales resultados concuerdan con lo expuesto en estudios previos (Guedes et al., 2013; Li et al., 2015; Tylka y Homan, 2015), los cuales dan cuenta de esta mayor asociación hacia factores de apariencia corporal. Estas motivaciones pueden estar asociadas a factores de carácter sociocultural e interpersonal, pero junto a ellas existen otras posibles causas que poseen un trasfondo social, como el hecho de que las mujeres experimentan una mayor presión hacia la belleza corporal y las formas de delgadez en comparación con los hombres. La influencia actual de los medios de comunicación, quienes hacen uso de una gran variedad de campañas publicitarias en las que mercadean el atractivo físico como sinónimo de salud. Ello ejerce un mayor impacto de estereotipos en las mujeres (Cagas, Torre y Manalastas, 2014; Kohlstedt, Weissbrod, Colangelo y Carter, 2013; Segar, Spruijt-Metz y Nolen-Hoeksema, 2006; Vartanian, Wharton y Green, 2012). Lo anterior permite interpretar que existe en las mujeres una mayor asociación con motivaciones más de carácter extrínsecas en comparación con las motivaciones de los hombres (Egli et al., 2011; Al Kubaisy et al., 2015; Louw et al., 2012; Molanorouzi et al., 2015).

Con respecto al tiempo que llevan realizando ejercicio físico de forma continua, las personas con más de un año mostraron resultados mayores asociados a motivos referentes a la sensación de afiliación, en comparación con las personas que llevan menos tiempo con dicha práctica. Estos resultados concuerdan con los mencionados en trabajos previos (Azofeifa, 2006; Guedes et al., 2013; Ramírez et al., 2013), en donde se manifiesta que las personas que llevan mayor tiempo realizando ejercicio físico lo relacionan con actividades más placenteras. Lo anterior puede ser explicado bajo la línea de estudios previos apoyados en el modelo transteórico del cambio, el cual indica que personas que encuentran en un estadio de mantenimiento hacia la práctica de ejercicio físico y han podido generar mecanismos sobre sus procesos cognitivos y de comportamiento incentivan motivos con características más autodeterminadas que les permite asociar la práctica permanente de ejercicio físico como un factor de disfrute (Azofeifa, 2006; Gómez-Tinoco, 2013; Jiménez-Castuera, Moreno-Navarrete, Leyton-Román y Claver-Rabaz, 2015; Sanclemente-Agualimpia y Arias-Henao, 2014; Sicilia et al., 2014).

Los resultados obtenidos también mostraron que motivos no autodeterminados, con características más extrínsecas, como es el caso del reconocimiento social, obtuvieron valores estadísticamente superiores en los sujetos que manifiestan realizar ejercicio físico de forma constante por más de un año, en comparación con quienes llevan seis meses a un año o menos tiempo en dicha práctica. Si bien tal manifestación podría ser más frecuente en sujetos con menor tiempo de práctica constante de ejercicio físico, los estudios realizados han mostrado tendencias similares hacia la manifestación de motivos con características más extrínsecas, de esta forma apoyados en la teoría multidimensional de los motivos hacia el ejercicio físico, permite interpretar la presencia de factores tanto extrínsecos como intrínsecos ya sea en la iniciación o en el mantenimiento de dicha práctica en las personas; a su vez, cabe la posibilidad de que el mayor reconocimiento social esté asociado a una necesidad incrementada de valoración o percepción por parte de la dinámica actual de la sociedad (Guedes et al., 2013; Moreno-Murcia, MarcosPardo y Huéscar, 2016; Sicilia et al., 2014). 


\section{Conclusiones}

Las mujeres parecen manifestar una mayor tendencia hacia la salud del cuerpo como uno de sus principales motivos para la práctica de ejercicio físico, en comparación con los hombres. Tal manifestación parece estar asociada a concepciones socioculturales arraigadas, en muchos casos producto de estereotipos, que traen como consecuencia un supuesto incremento de la presión asociada a la belleza y linealidad del cuerpo, fenómeno que se puede ver incrementado actualmente gracias al bombardeo de los medios de comunicación.

Una mayor afiliación como motivo para la práctica de ejercicio físico, en las personas que llevan mayor tiempo practicándolo de forma regular, puede ser producto de que estas se encuentran dentro de un estadio de mantenimiento basado en el modelo Transteórico del Cambio, lo cual puede generar en las personas mayores sensaciones de estabilidad, placer y disfrute sobre estas prácticas, permitiendo generar un mayor arraigo hacia el ejercicio físico como una práctica saludable que forme parte del diario vivir y no como una obligación, lujo o posibilidad de actividad.

El reconocimiento social fue un motivo manifestado por las personas con mayor tiempo practicando ejercicio físico de forma regular. Si bien este es un motivo más de características extrínsecas, que caracteriza a las personas principiantes, cabe la posibilidad de plantear que tanto los motivos intrínsecos como extrínsecos pueden influir, fluctuar y converger a lo largo del tiempo practicando ejercicio físico regular. De igual forma, no se puede obviar la necesidad de valoración u aceptación de agentes sociales, que pueden ser influenciados por los medios de comunicación, en la necesidad de generar un mayor reconocimiento social en las personas.

Los motivos que conducen a las personas a la realización de ejercicio físico a lo largo del tiempo parecen no mostrar una única dirección y, ante ello, la posibilidad de encontrar distintas motivaciones plantea el desafío de evolucionar constantemente en las estrategias hacia la adhesión de las personas en dicha práctica.

\section{Referencias}

Al Kubaisy, W, Mohamad, M., Ismail, Z., \& Abdullah, N. N. (2015). Gender Differences: Motivations for performing physical exercise among adults in Shah Alam. Procedia - Social and Behavioral Sciences, 202, 522-530. doi: https://doi.org/10.1016/j.sbspro.2015.08.181

Azofeifa, E. G. (2006). Motivos de participación y satisfacción en la actividad física, el ejercicio físico y el deporte. Revista MHSalud, 3(1), 1-16. Recuperado de http://www.revistas.una.ac.cr/index.php/ mhsalud/article/view/318

Ball, J. W., Bice, M. R., \& Parry, T. (2014). Adults' Motivation for Physical Activity: Differentiating Motives for Exercise, Sport, and Recreation. Recreational Sports Journal, 38(2), 130-142. Retrieved from http:// search.ebscohost.com/login.aspx?direct=true\&db=s3h\&AN=99683652\&lang=es\&site=ehost-live

Cagas, J., Torre, B., \& Manalastas, E. J. (2014). Pampapayat, Para Lumakas, To Be Healthy: Exploring Filipino Motives For Exercise. Journal of psyChology, 47(1), 145-161. Retrieved from http://pages.upd.edu.ph/sites/default/files/ejmanalastas/files/ cagas_torre_manalastas_2014_pjp_exercise_motives.pdf

Egli, T., Bland, H. W., Melton, B. F., \& Czech, D. R. (2011). Influence of age, sex, and race on college students' exercise motivation of physical activity. Journal of American College Health, 59(5), 399-406. doi: https://doi.org/10.1080/07448481.2010.513074

Folgar, M. I., Boubeta, A. R., Cristobal, R. V., y Zamácola, F. S. (2012). Motivaciones para la práctica de deporte federado y del piragüismo en alumnos de primaria y secundaria. Retos: Nuevas Perspectivas de Educación Física, Deporte y Recreación, 21(21), 19-24. Recuperado de http://ezproxy.library.yorku.ca/login?url=http://search.ebscohost.com/login.aspx? direct=true \&db=sph\&AN=85719081\&site=ehost-live 
Gómez-Tinoco, M. C. (2013). Comparison of the Stages of Change for Physical Activity and Physical Exercise in Brazilian and Catalan University Students. Revista Científica General José María Córdova, 11(12), 173-198. Retrieved from http://www.scielo.org.co/pdf/recig/v11n12/v11n12a09.pdf

Guedes, D. P., Legnani, R. F. S., \& Legnani, E. (2013). Exercise motives in a sample of Brazilian university students. Motriz. Rio Claro, 19(3), 590-596. doi: https://doi.org/10.1590/S1980-65742013000300009

Gutiérrez, T., Raich, R. M., Sánchez, D., y Deus, J. (2003). Instrumentos de evaluación de Psicología de la Salud. Madrid: Alianza Editorial.

Jiménez-Castuera, R., Moreno-Navarrete, B., Leyton-Román, M., y Claver-Rabaz, F. (2015). Motivación y estadios de cambio para el ejercicio físico en adolescentes. Revista Latinoamericana de Psicología, 47, 196-204. doi: https://doi.org/dx.doi.org/10.1016/j.rlp.2014.11.001 0120-0534/

Jiménez-Torres, M. G., Godoy-Izquierdo, D., y Godoy García, J. F. (2012). Relación entre los motivos para la práctica físico-deportiva y las experiencias de flujo en jóvenes: diferencias en función del sexo. Universitas Psychologica, 11(3), 909-920. Recuperado de http://search.ebscohost.com/login.aspx? direct=true\&db=a9h\&AN=89172525\&lang=es\&site=ehost-live

Kohlstedt, S. S., Weissbrod, C. S., Colangelo, A. M., \& Carter, M. M. (2013). Psychological Factors Influencing Exercise Adherence among Females. Psychology, 4(12), 917-923. doi: https://doi.org/10.4236/ psych.2013.412132

Li, C., Zayed, K., Muazzam, A., Li, M., Cheng, J., \& Chen, A. (2015). Motives for Exercise in Undergraduate Muslim Women and Men in Oman and Pakistan Compared to the United States. Sex Roles, 72(1-2), 68-84. Retrieved from http://10.0.3.239/s11199-014-0435-z

Louw, A. J., Van-Biljon, A., \& Mugandani, S. C. (2012). Exercise motivation and barriers among men and women of different age groups. African Journal for Physical, Health Education, Recreation \& Dance, 18(4), 759-768. Retrieved from http://search.ebscohost.com/login.aspx?

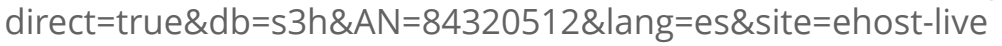

Molanorouzi, K., Khoo, S., \& Morris, T. (2015). Motives for adult participation in physical activity: type of activity, age, and gender. BMC Public Health, 15(1), 66. doi: https://doi.org/10.1186/ s12889-015-1429-7

Moreno-Collazos, J. E., y Cruz-Bermúdez, H. F. (2015). Asociación entre los motivos y la práctica del ejercicio en participantes de un programa de actividad física laboral. Rev. Fac. Med., 63(4), 609-615. doi: https://doi.org/10.15446/revfacmed.v63.n4.49238

Moreno-Murcia, J. A., Marcos-Pardo, P. J., y Huéscar, E. (2016). Motivos de Práctica Físico-Deportiva en Mujeres: Diferencias entre Practicantes y no Practicantes. Revista de Psicología Del Deporte, 25(1), 35-41. Recuperado de http://www.rpd-online.com/article/viewFile/v25-n1-moreno-murcia-marcosetal/Moreno_Murcia_Marcosetal

Navas, L., y Soriano, J. A. (2016). Análisis de los Motivos para Practicar o No Actividades Físicas Extracurriculares y su Relación con el Autoconcepto Físico en Estudiantes Chilenos. Revista Iberoamericana de Psicología Del Ejercicio Y El Deporte, 11(1), 69-76. Recuperado de https:// rua.ua.es/dspace/bitstream/10045/52609/1/2016_Navas_Soriano_RIPED.pdf

Pérez-muñoz, S., y Sánchez-vegas, M. (2015). Motivos de Participación y Satisfacción en Actividades Cooperativas y No Competitivas en el Medio Natural. Ágora Para La EF Y El Deporte, 17(3), 284-299. Recuperado de http://agora-revista.blogs.uva.es/files/2015/12/agora_17_3f_perez_et_sanchez.pdf

Ramírez, M. J., Godoy-Izquierdo, D., Vázquez, M. L., Lara, R., Navarrón, E., Vélez, M., ... Torres, A. G. (2013). Motivos de práctica de ejercicio físico en adultos jóvenes con una imagen corporal positiva. Anuario de Psicología Clínica Y de La Salud, 9, 93-96. Recuperado de http://institucional.us.es/apcs/doc/ APCS_9_esp_93-96.pdf

Sanclemente-Agualimpia, Y. A., y Arias-Henao, M. (2014). Factores motivacionales que influyen en la adherencia a la actividad física en los usuarios del Gimnasio Zona Fitness. Revista de Educación 
Física, 3(1), 2322-9411. Recuperado de https://aprendeenlinea.udea.edu.co/revistas/index.php/ viref/article/viewFile/19050/16277

Segar, M., Spruijt-Metz, D., \& Nolen-Hoeksema, S. (2006). Go Figure? Body-Shape Motives are Associated with Decreased Physical Activity Participation Among Midlife Women. Sex Roles, 54(3/4). doi: https:// doi.org/10.1007/s11199-006-9336-5

Sicilia, Á., González-Cutre, D., Artés, E. M., Orta, A., Casimiro, A. J., y Ferriz, R. (2014). Motivos de los ciudadanos para realizar ejercicio físico: un estudio desde la teoría de la autodeterminación. Revista Latinoamericana de Psicología, 46(2), 83-91. doi: https://doi.org/10.1016/S0120-0534(14)70011-1

Teixeira, P. J., Carraça, E. V, Markland, D., Silva, M. N., \& Ryan, R. M. (2012). Exercise, physical activity, and self-determination theory: A systematic review. International Journal of Behavioral Nutrition and Physical Activity, 9(1), 78. doi: https://doi.org/10.1186/1479-5868-9-78

Tylka, T. L., \& Homan, K. J. (2015). Exercise motives and positive body image in physically active college women and men: Exploring an expanded acceptance model of intuitive eating. Body Image, 15, 9097. doi: https://doi.org/10.1016/j.bodyim.2015.07.003

Usán-Supervía, P., Salavera-Bordás, C., Murillo-Lorente, V., y Megías-Abad, J. J. (2016). Relación entre motivación, compromiso y autoconcepto en adolescentes: estudio con futbolistas. Cuadernos de Psicología del Deporte 16(1), 199-210. Recuperado de http://revistas.um.es/cpd/article/ download/254561/192191

Vartanian, L. R., Wharton, C. M., \& Green, E. B. (2012). Appearance vs. health motives for exercise and for weight loss. Psychology of Sport \& Exercise, 13, 251-256. doi: https://doi.org/10.1016/ j.psychsport.2011.12.005 